\title{
The 1:3 Internal Resonance of Landing Gear Nonlinear Vibrating System
}

$$
\text { Yan-Ying Zhao }{ }^{\text {a, }} \text {, Fang-Gui Yu and Dunke Song }
$$

School of Aircraft Engineering, Nanchang Hangkong University, Nanchang 330063, China

ayanyingzhao@nchu.edu.cn

${ }^{*}$ Corresponding author

Keywords: Internal resonance, Landing gear system, delayed feedback control.

Abstract. In this paper, a two-degree of nonlinear vibrating system of landing gear is studied. A delayed velocity feedback is applied to suppress the vibration of the landing gear structure. The dynamical behavior of landing gear system is investigated when the 1:3 internal resonance and primary external resonance occur simultaneously. Two cases of primary resonance are analyzed when the external frequency nears to the first and second mode frequency of the system. The effects of delayed feedback gain and time delay on the vibration of the landing gear system are investigated. The results show that the suppression ability is improved when the value of delayed feedback gain is increased. However, the unstable range of the system is enlarged with the value of delayed feedback gain increases. Another result is that the amplitude of the landing gear system can be suppressed to a minimum value if the time delay is chosen properly.

\section{Introduction}

The fundamental function of landing gear structure is to reduce the external excitation during landing and taxiing. The aircraft taxiing track irregularity results in the vibration of landing gear structure. This kind of vibration may lead to lower aircraft life, poor stability, difficult operation and lower passenger comfort. In 1937, landing gear system is modeled as a linear spring damper system by F. Michael modeled ${ }^{[1]}$. K.Schlaefke ${ }^{[2]}$ optimize the linear spring damper landing gear system based his previous experimental study. K. Schlaefke and W. Kochanowsky ${ }^{[3]}$ simplify the tire into a linear spring while neglecting the damping and impact of load during landing and taxiing. E. Marquard and W. Meyer Capplengon ${ }^{[4]}$ investigate the nonlinear spring damper landing gear system. W. Flugge ${ }^{[5]}$ analyze the linear spring damper system in detail, and also investigate the nonlinear buffer structure. His results show that nonlinear system can simulate the actual motion state of aircraft better during landing and taxiing. I.Ross ${ }^{[6]}$ discussed the application of active control technology in the semi-active control field in some military landing gear system. In fact, aircraft landing gear system is actually a nonlinear vibrating system composed of nonlinear absorber, tire and bars. In the present paper, the authors used the delayed feedback control to suppress the vibration of nonlinear landing gear vibrating system. The results show the performance of vibration suppression is perfect.

\section{The Differential Equation of Landing Gear System}

A two-degree of freedom nonlinear landing gear vibrating system with delayed feedback control is shown in Fig. 1. The differential equation of vibration with the delayed feedback control is

$$
\begin{aligned}
& \mathrm{m}_{1} \ddot{\mathrm{y}}+\mathrm{c}_{1}\left(\dot{\mathrm{y}}_{1}-\dot{\mathrm{y}}_{2}\right)+\mathrm{k}_{1}\left(\mathrm{y}_{1}-\mathrm{y}_{2}\right)+\delta_{0}\left(\mathrm{y}_{1}-\mathrm{y}_{2}\right)^{2}+\delta_{1}\left(\mathrm{y}_{1}-\mathrm{y}_{2}\right)^{3}+\mathrm{g} \dot{\mathrm{y}}(\mathrm{t}-\tau)=0 \\
& \mathrm{~m}_{2} \ddot{\mathrm{y}}_{2}-\mathrm{c}_{1}\left(\dot{\mathrm{y}}_{1}-\dot{\mathrm{y}}_{2}\right)-\mathrm{k}_{1}\left(\mathrm{y}_{1}-\mathrm{y}_{2}\right)-\delta_{0}\left(\mathrm{y}_{1}-\mathrm{y}_{2}\right)^{2}-\delta_{1}\left(\mathrm{y}_{1}-\mathrm{y}_{2}\right)^{3}-\mathrm{g} \dot{\mathrm{y}}(\mathrm{t}-\tau)+\mathrm{k}_{2} \mathrm{y}_{2}+\mathrm{c}_{2} \dot{\mathrm{y}}_{2}=\mathrm{F} \cos (\omega \mathrm{t})
\end{aligned}
$$




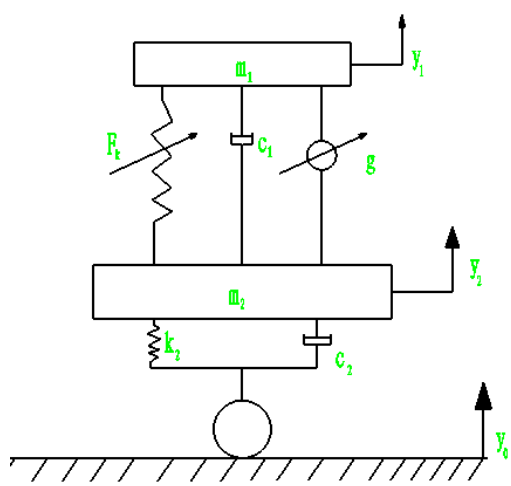

Fig. 1: A two-degree of freedom nonlinear landing gear vibrating system with delayed feedback control

In this system, $m_{1}$ is the mass of the buffer structure of aircraft landing gear, $m_{2}$ is the mass of the machine wheel and wheel set. $\mathrm{y}_{1}$ and $\mathrm{y}_{2}$ are represent the vertical displacement of landing gear and wheel set. The relationship between force and displacement satisfies the condition of $\mathrm{F}_{\mathrm{k}}=\mathrm{k}_{1} \mathrm{y}+\delta_{0} \mathrm{y}^{2}+\delta_{1} \mathrm{y}^{3}, \mathrm{k}_{1}$ and $\mathrm{k}_{2}$ are the linear spring stiffness coefficient, $\delta_{0}$ and $\delta_{1}$ are quadratic and cubic nonlinear spring stiffness coefficients respectively. $c_{1}$ and $c_{2}$ are the linear damping coefficients of the landing gear and wheel set. The landing gear system is excited by road irregularity external excitation $\mathrm{y}_{0}=\mathrm{F} \cos (\Omega \mathrm{t}), \mathrm{F}$ is the amplitude corresponding to the external excitation, $\Omega$ is the frequency of external excitation, $\mathrm{t}$ is the time, and $(\cdot)=\mathrm{d}() / \mathrm{dt} . \mathrm{g}$ is the linear damping feedback gain of the delayed feedback control signal $\mathrm{F}_{\mathrm{S}}=\mathrm{g} \dot{\mathrm{y}}(\mathrm{t}-\tau), \tau$ is the time delay, and $\dot{\mathrm{y}}(\mathrm{t}-\tau)=\dot{\mathrm{y}}_{\tau}$.

Eqs. (1) and (2) can be expressed as

$$
\begin{gathered}
{\left[\begin{array}{cc}
\mathrm{m}_{1}+\mathrm{r}_{1}^{2} \mathrm{~m}_{2} & 0 \\
0 & \mathrm{~m}_{1}+\mathrm{r}_{2}^{2} \mathrm{~m}_{2}
\end{array}\right]\left\{\begin{array}{l}
\ddot{\mathrm{q}}_{1} \\
\ddot{\mathrm{q}}_{2}
\end{array}\right\}+\left[\begin{array}{cc}
\mathrm{k}_{1}-2 \mathrm{r}_{1} \mathrm{k}_{1}+\mathrm{r}_{1}^{2}\left(\mathrm{k}_{1}+\mathrm{k}_{2}\right) & 0 \\
0 & \mathrm{k}_{1}-2 \mathrm{r}_{2} \mathrm{k}_{1}+\mathrm{r}_{2}^{2}\left(\mathrm{k}_{1}+\mathrm{k}_{2}\right)
\end{array}\right]\left\{\begin{array}{l}
\mathrm{q}_{1} \\
\mathrm{q}_{2}
\end{array}\right\}} \\
+\varepsilon\left\{\begin{array}{l}
\left(1-\mathrm{r}_{1}\right)\left(\dot{\mathrm{q}}_{1 \tau}+\dot{\mathrm{q}}_{2 \tau}\right)+\left(1-\mathrm{r}_{1}\right) \delta_{0}\left(\mathrm{q}_{1}+\mathrm{q}_{2}-\mathrm{r}_{1} \mathrm{q}_{1}-\mathrm{r}_{2} \mathrm{q}_{2}\right)^{2}+\left(1-\mathrm{r}_{1}\right) \delta_{1}\left(\mathrm{q}_{1}+\mathrm{q}_{2}-\mathrm{r}_{1} \mathrm{q}_{1}-\mathrm{r}_{2} \mathrm{q}_{2}\right)^{3} \\
\left(1-\mathrm{r}_{2}\right)\left(\dot{\mathrm{q}}_{1 \tau}+\dot{\mathrm{q}}_{2 \tau}\right)+\left(1-\mathrm{r}_{1}\right) \delta_{0}\left(\mathrm{q}_{1}+\mathrm{q}_{2}-\mathrm{r}_{1} \mathrm{q}_{1}-\mathrm{r}_{2} \mathrm{q}_{2}\right)^{2}+\left(1-\mathrm{r}_{2}\right) \delta_{1}\left(\mathrm{q}_{1}+\mathrm{q}_{2}-\mathrm{r}_{1} \mathrm{q}_{1}-\mathrm{r}_{2} \mathrm{q}_{2}\right)^{3}
\end{array}\right\} \\
+\varepsilon\left[\begin{array}{cc}
\mathrm{c}_{1}-2 \mathrm{r}_{1} \mathrm{c}_{1}+\mathrm{r}_{1}^{2}\left(\mathrm{c}_{1}+\mathrm{c}_{2}\right) & \mathrm{c}_{1}-\mathrm{r}_{1} \mathrm{c}_{1}-\mathrm{r}_{2} \mathrm{c}_{1}+\mathrm{r}_{1} \mathrm{r}_{2}\left(\mathrm{c}_{1}+\mathrm{c}_{2}\right) \\
\mathrm{c}_{1}-\mathrm{r}_{1} \mathrm{c}_{1}-\mathrm{r}_{2} \mathrm{c}_{1}+\mathrm{r}_{1} \mathrm{r}_{2}\left(\mathrm{c}_{1}+\mathrm{c}_{2}\right) & \mathrm{c}_{1}-2 \mathrm{r}_{2} \mathrm{c}_{1}+\mathrm{r}_{2}^{2}\left(\mathrm{c}_{1}+\mathrm{c}_{2}\right)
\end{array}\right]\left\{\begin{array}{l}
\dot{\mathrm{q}}_{1} \\
\dot{\mathrm{q}}_{2}
\end{array}\right\}=\varepsilon\left\{\begin{array}{l}
\mathrm{r}_{1} \mathrm{f} \cos (\omega \mathrm{t}) \\
\mathrm{r}_{2} \mathrm{f} \cos (\omega \mathrm{t})
\end{array}\right\}
\end{gathered}
$$

where $\omega_{1}$ and $\omega_{2}$ are the first and second modal frequency of the vibrating system, $\mathrm{r}_{1}=1-\frac{\mathrm{m}_{1}}{\mathrm{k}_{1}} \omega_{1}^{2}$, $\mathrm{r}_{2}=1-\frac{\mathrm{m}_{1}}{\mathrm{k}_{1}} \omega_{2}^{2}, \mathrm{c}_{1}=\varepsilon \mathrm{c}_{1}, \mathrm{c}_{2}=\varepsilon \mathrm{c}_{2}, \mathrm{~g}=\varepsilon \mathrm{g}, \mathrm{F}=\varepsilon \mathrm{f},\left[\begin{array}{l}\mathrm{x}_{1} \\ \mathrm{x}_{2}\end{array}\right]=\left[\begin{array}{ll}1 & 1 \\ \mathrm{r}_{1} & \mathrm{r}_{2}\end{array}\right]\left[\begin{array}{l}\mathrm{q}_{1} \\ \mathrm{q}_{2}\end{array}\right]$.

\section{Perturbation Analysis}

In this section, the method of multiple scales is applied to obtain the second-order approximate solutions of Eqs. (3) using the following form

$$
\begin{aligned}
& \mathrm{q}_{1}(\mathrm{t}, \varepsilon)=\mathrm{q}_{11}\left(\mathrm{~T}_{0}, \mathrm{~T}_{1}, \cdots\right)+\varepsilon \mathrm{q}_{12}\left(\mathrm{~T}_{0}, \mathrm{~T}_{1},+\cdots\right)+\cdots \\
& \mathrm{q}_{2}(\mathrm{t}, \varepsilon)=\mathrm{q}_{21}\left(\mathrm{~T}_{0}, \mathrm{~T}_{1}, \cdots\right)+\varepsilon \mathrm{q}_{22}\left(\mathrm{~T}_{0}, \mathrm{~T}_{1},+\cdots\right)+\cdots \\
& \mathrm{q}_{1 \tau}=\mathrm{q}_{11 \tau}\left(\mathrm{T}_{0}-\tau, \mathrm{T}_{1}, \mathrm{~T}_{2}, \cdots\right)-\varepsilon \tau \mathrm{D}_{1} \mathrm{q}_{11 \tau}\left(\mathrm{T}_{0}-\tau, \mathrm{T}_{1}, \mathrm{~T}_{2}, \cdots\right)+\varepsilon \mathrm{D}_{1} \mathrm{q}_{12 \tau}\left(\mathrm{T}_{0}-\tau, \mathrm{T}_{1}, \mathrm{~T}_{2}, \cdots\right)
\end{aligned}
$$


$+\frac{\varepsilon^{2} \tau^{2}}{2} \mathrm{D}_{1}^{2} \mathrm{q}_{11 \tau}\left(\mathrm{T}_{0}-\tau, \mathrm{T}_{1}, \mathrm{~T}_{2}, \cdots\right)-\varepsilon^{2} \tau \mathrm{D}_{1} \mathrm{q}_{12 \tau}\left(\mathrm{T}_{0}-\tau, \mathrm{T}_{1}, \mathrm{~T}_{2}, \cdots\right)+\cdots$

$\mathrm{q}_{2 \tau}=\mathrm{q}_{21 \tau}\left(\mathrm{T}_{0}-\tau, \mathrm{T}_{1}, \mathrm{~T}_{2}, \cdots\right)-\varepsilon \tau \mathrm{D}_{1} \mathrm{q}_{21 \tau}\left(\mathrm{T}_{0}-\tau, \mathrm{T}_{1}, \mathrm{~T}_{2}, \cdots\right)+\varepsilon \mathrm{D}_{1} \mathrm{q}_{22 \tau}\left(\mathrm{T}_{0}-\tau, \mathrm{T}_{1}, \mathrm{~T}_{2}, \cdots\right)$

$+\frac{\varepsilon^{2} \tau^{2}}{2} \mathrm{D}_{1}^{2} \mathrm{q}_{21 \tau}\left(\mathrm{T}_{0}-\tau, \mathrm{T}_{1}, \mathrm{~T}_{2}, \cdots\right)-\varepsilon^{2} \tau \mathrm{D}_{1} \mathrm{q}_{22 \tau}\left(\mathrm{T}_{0}-\tau, \mathrm{T}_{1}, \mathrm{~T}_{2}, \cdots\right)+\cdots$

Substituting Eqs. (4)-(7) into Eq. (3), and equating coefficients of like powers of $\varepsilon$ yield $\varepsilon^{0}$

$D_{0}^{2} q_{11}+\omega_{1}^{2} q_{11}=0$

$D_{0}^{2} q_{21}+\omega_{2}^{2} q_{21}=0$

$\varepsilon^{1}:$

$\left(\mathrm{m}_{1}+\mathrm{r}_{1}^{2} \mathrm{~m}_{2}\right) \mathrm{D}_{0}^{2} \mathrm{q}_{12}+\left[\mathrm{k}_{1}-2 \mathrm{r}_{1} \mathrm{k}_{1}+\mathrm{r}_{1}^{2}\left(\mathrm{k}_{1}+\mathrm{k}_{2}\right)\right] \mathrm{q}_{12}=-2\left(\mathrm{~m}_{1}+\mathrm{r}_{1}^{2} \mathrm{~m}_{2}\right) \mathrm{D}_{0} \mathrm{D}_{1} \mathrm{q}_{11}-\left[\mathrm{c}_{1}-\right.$

$\left.2 \mathrm{r}_{1} \mathrm{c}_{1}+\mathrm{r}_{1}^{2}\left(\mathrm{c}_{1}+\mathrm{c}_{2}\right)\right] \mathrm{D}_{0} \mathrm{q}_{11}-\left[\mathrm{c}_{1}-\mathrm{r}_{1} \mathrm{c}_{1}-\mathrm{r}_{2} \mathrm{c}_{1}+\mathrm{r}_{1} \mathrm{r}_{2}\left(\mathrm{c}_{1}+\mathrm{c}_{2}\right] \mathrm{D}_{0} \mathrm{q}_{12}-\left(1-\mathrm{r}_{1}\right)\left[\mathrm{g}\left(\mathrm{D}_{0} \mathrm{q}_{11 \tau}\right.\right.\right.$

$\left.\left.+\mathrm{D}_{0} \mathrm{q}_{12 \tau}\right)+\delta_{0}\left(\mathrm{q}_{1}+\mathrm{q}_{2}-\mathrm{r}_{1} \mathrm{q}_{1}-\mathrm{r}_{2} \mathrm{q}_{2}\right)^{2}+\delta_{1}\left(\mathrm{q}_{1}+\mathrm{q}_{2}-\mathrm{r}_{1} \mathrm{q}_{1}-\mathrm{r}_{2} \mathrm{q}_{2}\right)^{3}\right]+\mathrm{r}_{1} \mathrm{f} \cos (\omega \mathrm{t})$

$\left(\mathrm{m}_{1}+\mathrm{r}_{2}^{2} \mathrm{~m}_{2}\right) \mathrm{D}_{0}^{2} \mathrm{q}_{22}+\left[\mathrm{k}_{1}-2 \mathrm{r}_{2} \mathrm{k}_{1}+\mathrm{r}_{2}^{2}\left(\mathrm{k}_{1}+\mathrm{k}_{2}\right)\right] \mathrm{q}_{22}=-2\left(\mathrm{~m}_{1}+\mathrm{r}_{2}^{2} \mathrm{~m}_{2}\right) \mathrm{D}_{0} \mathrm{D}_{1} \mathrm{q}_{21}-\left[\mathrm{c}_{1}-\mathrm{r}_{1} \mathrm{c}_{1}-\right.$

$\left.\mathrm{r}_{2} \mathrm{c}_{1}+\mathrm{r}_{1} \mathrm{r}_{2}\left(\mathrm{c}_{1}+\mathrm{c}_{2}\right)\right] \mathrm{D}_{0} \mathrm{q}_{11}-\left[\mathrm{c}_{1}-2 \mathrm{r}_{2} \mathrm{c}_{1}+\mathrm{r}_{2}^{2}\left(\mathrm{c}_{1}+\mathrm{c}_{2}\right] \mathrm{D}_{0} \mathrm{q}_{21}-\left(1-\mathrm{r}_{2}\right)\left[\mathrm{g}\left(\mathrm{D}_{0} \mathrm{q}_{11 \tau}+\mathrm{D}_{0} \mathrm{q}_{12 \tau}\right)\right.\right.$

$\left.+\delta_{0}\left(\mathrm{q}_{1}+\mathrm{q}_{2}-\mathrm{r}_{1} \mathrm{q}_{1}-\mathrm{r}_{2} \mathrm{q}_{2}\right)^{2}+\delta_{1}\left(\mathrm{q}_{1}+\mathrm{q}_{2}-\mathrm{r}_{1} \mathrm{q}_{1}-\mathrm{r}_{2} \mathrm{q}_{2}\right)^{3}\right]+\mathrm{r}_{2} \mathrm{f} \cos (\omega \mathrm{t})$

Where $\omega_{1}^{2}=\frac{\mathrm{k}_{1}-2 \mathrm{r}_{1} \mathrm{k}_{1}+\mathrm{r}_{1}^{2}\left(\mathrm{k}_{1}+\mathrm{k}_{2}\right)}{\mathrm{m}_{1}+\mathrm{r}_{1}^{2} \mathrm{~m}_{2}}, \omega_{2}^{2}=\frac{\mathrm{k}_{1}-2 \mathrm{r}_{2} \mathrm{k}_{1}+\mathrm{r}_{2}^{2}\left(\mathrm{k}_{1}+\mathrm{k}_{2}\right)}{\mathrm{m}_{1}+\mathrm{r}_{2}^{2} \mathrm{~m}_{2}}$.

The solutions of Eqs. (8) and (9) can be expressed as

$$
\begin{aligned}
& \mathrm{q}_{11}=\mathrm{A}\left(\mathrm{T}_{1}\right) \mathrm{e}^{\mathrm{i} \omega_{1} \mathrm{~T}_{0}}+\overline{\mathrm{A}}\left(\mathrm{T}_{1}\right) \mathrm{e}^{-\mathrm{i} \omega_{1} \mathrm{~T}_{0}} \\
& \mathrm{q}_{21}=\mathrm{B}\left(\mathrm{T}_{1}\right) \mathrm{e}^{\mathrm{i} \omega_{2} \mathrm{~T}_{0}}+\overline{\mathrm{B}}\left(\mathrm{T}_{1}\right) \mathrm{e}^{-\mathrm{i} \omega_{2} \mathrm{~T}_{0}}
\end{aligned}
$$

Substituting Eqs. (12)-(13) into Eqs. (10)-(11), we can obtain

$$
\begin{aligned}
& \mathrm{D}_{0}^{2} \mathrm{q}_{12}+\omega_{1}^{2} \mathrm{q}_{12}=\frac{1}{\mathrm{~m}_{1}+\mathrm{r}_{1}^{2} \mathrm{~m}_{2}}\left\{\mathrm{k}_{1} \mathrm{e}^{\mathrm{i} \omega_{1} \mathrm{~T}_{0}}+\mathrm{k}_{2} \mathrm{e}^{\mathrm{i} \omega_{2} \mathrm{~T}_{0}}+\mathrm{k}_{3} \mathrm{e}^{2 \mathrm{i} \omega_{1} \mathrm{~T}_{0}}+\mathrm{k}_{4} \mathrm{e}^{\mathrm{i}\left(\omega_{1}+\omega_{2}\right) \mathrm{T}_{0}}+\mathrm{k}_{5} \mathrm{e}^{\mathrm{i}\left(\omega_{2}-\omega_{1}\right) \mathrm{T}_{0}}\right. \\
& \mathrm{k}_{6} \mathrm{e}^{2 \mathrm{i} \omega_{2} \mathrm{~T}_{0}}+\mathrm{k}_{7} \mathrm{e}^{3 \mathrm{i} \omega_{1} \mathrm{~T}_{0}}+\mathrm{k}_{8} \mathrm{e}^{\mathrm{i}\left(2 \omega_{1}+\omega_{2}\right) \mathrm{T}_{0}}+\mathrm{k}_{9} \mathrm{e}^{\mathrm{i}\left(\omega_{2}-2 \omega_{1}\right) \mathrm{T}_{0}}+\mathrm{k}_{10} \mathrm{e}^{\mathrm{i}\left(2 \omega_{2}+\omega_{1}\right) \mathrm{T}_{0}}+\mathrm{k}_{11} \mathrm{e}^{\mathrm{i}\left(2 \omega_{2}-\omega_{1}\right) \mathrm{T}_{0}}+\mathrm{k}_{12} \mathrm{e}^{3 \mathrm{i} \omega_{2} \mathrm{~T}_{0}} \\
& \left.+\mathrm{k}_{13} \mathrm{e}^{\mathrm{i} \omega_{1}\left(\mathrm{~T}_{0}-\tau\right)}+\mathrm{k}_{14} \mathrm{e}^{\mathrm{i} \omega_{2}\left(\mathrm{~T}_{0}-\tau\right)}+\mathrm{k}_{15} \mathrm{e}^{\mathrm{i} \mathrm{T}_{0}}+\mathrm{k}_{16}+\mathrm{cc}\right\} \\
& \mathrm{D}_{0}^{2} \mathrm{q}_{22}+\omega_{2}^{2} \mathrm{q}_{22}=\frac{1}{\mathrm{~m}_{1}+\mathrm{r}_{2}^{2} \mathrm{~m}_{2}}\left\{\mathrm{k}_{17} \mathrm{e}^{\mathrm{i} \omega_{1} \mathrm{~T}_{0}}+\mathrm{k}_{18} \mathrm{e}^{\mathrm{i} \omega_{2} \mathrm{~T}_{0}}+\mathrm{k}_{19} \mathrm{e}^{2 \mathrm{i} \omega_{1} \mathrm{~T}_{0}}+\mathrm{k}_{20} \mathrm{e}^{\mathrm{i}\left(\omega_{1}+\omega_{2}\right) \mathrm{T}_{0}}+\mathrm{k}_{21} \mathrm{e}^{\mathrm{i}\left(\omega_{2}-\omega_{1}\right) \mathrm{T}_{0}}\right. \\
& \mathrm{k}_{22} \mathrm{e}^{2 \mathrm{i} \omega_{2} \mathrm{~T}_{0}}+\mathrm{k}_{23} \mathrm{e}^{3 \mathrm{i} \omega_{1} \mathrm{~T}_{0}}+\mathrm{k}_{24} \mathrm{e}^{\mathrm{i}\left(2 \omega_{1}+\omega_{2}\right) \mathrm{T}_{0}}+\mathrm{k}_{25} \mathrm{e}^{\mathrm{i}\left(\omega_{2}-2 \omega_{1}\right) \mathrm{T}_{0}}+\mathrm{k}_{26} \mathrm{e}^{\mathrm{i}\left(2 \omega_{2}+\omega_{1}\right) \mathrm{T}_{0}}+\mathrm{k}_{27} \mathrm{e}^{\mathrm{i}\left(2 \omega_{2}-\omega_{1}\right) \mathrm{T}_{0}}+\mathrm{k}_{28} \mathrm{e}^{3 i \omega_{2} \mathrm{~T}_{0}} \\
& \left.+\mathrm{k}_{29} \mathrm{e}^{\mathrm{i} \omega_{1}\left(\mathrm{~T}_{0}-\tau\right)}+\mathrm{k}_{30} \mathrm{e}^{\mathrm{i} \omega_{2}\left(\mathrm{~T}_{0}-\tau\right)}+\mathrm{k}_{31} \mathrm{e}^{\mathrm{i} \mathrm{T}_{0}}+\mathrm{k}_{32}+\mathrm{cc}\right\}
\end{aligned}
$$

where cc denotes the complex conjugate terms, and $\mathrm{k}_{1}-\mathrm{k}_{32}$ are the coefficients, the express of $\mathrm{k}_{1}$ $\mathrm{k}_{32}$ is omitted due to space limitations. 
In the following section, primary resonance and 1:3 internal resonance is considered. The nearness of the internal resonance is represented by a detuning parameter $\sigma_{3}$ defined as $\omega_{2}=3 \omega_{1}+\varepsilon \sigma_{3}$. Two cases of external resonance are analyzed. The first case is the external excitation frequency nears to the first mode frequency of the system. The second case is the external excitation frequency nears to the second mode frequency of the system.

For the first case of $\omega \rightarrow \omega_{1}$, to describe the nearness of the external resonance quantitatively, a detuning parameter $\sigma_{1}$ is introduced as $\omega=\omega_{1}+\varepsilon \sigma_{1}$. Setting the coefficients of the secular terms to zero into Eqs. (14)-(15), and yield the solvability conditions which are given by

$$
\begin{aligned}
& \mathrm{D}_{1} \mathrm{~A}=\frac{1}{2\left(\mathrm{~m}_{1}+\mathrm{r}_{1}^{2} \mathrm{~m}_{2}\right) i \omega_{1}}\left\{-\left[\mathrm{c}_{1}-2 \mathrm{r}_{1} \mathrm{c}_{1}+\mathrm{r}_{1}^{2}\left(\mathrm{c}_{1}+\mathrm{c}_{2}\right)\right] i \omega_{1} \mathrm{~A}-6 \delta_{1}\left(1-\mathrm{r}_{1}\right)^{2}\left(1-\mathrm{r}_{2}\right)^{2} \mathrm{~B} \overline{\mathrm{B}} \mathrm{A}\right. \\
& \left.-3 \delta_{1}\left(1-\mathrm{r}_{1}\right)^{4} \mathrm{~A}^{2} \overline{\mathrm{A}}-3 \delta_{1}\left(1-\mathrm{r}_{1}\right)^{3}\left(1-\mathrm{r}_{2}\right) \overline{\mathrm{A}}^{2} \mathrm{Be} \mathrm{e}^{\mathrm{i} \mathrm{\sigma}_{3} \mathrm{~T}_{1}}-\mathrm{i}\left(1-\mathrm{r}_{1}\right) g \omega_{1} A \mathrm{e}^{-\mathrm{i} \omega_{1} \tau}+\frac{1}{2} \mathrm{r}_{1} \mathrm{fe}^{\mathrm{i} \sigma_{1} \mathrm{~T}_{1}}\right\} \\
& \mathrm{D}_{1} \mathrm{~B}=\frac{1}{2\left(\mathrm{~m}_{1}+\mathrm{r}_{2}^{2} \mathrm{~m}_{2}\right) i \omega_{2}}\left\{-\left[\mathrm{c}_{1}-2 \mathrm{r}_{2} \mathrm{c}_{1}+\mathrm{r}_{2}^{2}\left(\mathrm{c}_{1}+\mathrm{c}_{2}\right)\right] i \omega_{2} \mathrm{~B}-6 \delta_{1}\left(1-\mathrm{r}_{2}\right)^{2}\left(1-\mathrm{r}_{1}\right)^{2} \mathrm{~A} \overline{\mathrm{A}} \mathrm{B}\right. \\
& \left.-3 \delta_{1}\left(1-\mathrm{r}_{2}\right)^{4} \mathrm{~B}^{2} \overline{\mathrm{B}}-\delta_{1}\left(1-\mathrm{r}_{1}\right)^{3}\left(1-\mathrm{r}_{2}\right) \mathrm{A}^{3} \mathrm{e}^{-\mathrm{i} \sigma_{3} \mathrm{~T}_{1}}-\mathrm{i}\left(1-\mathrm{r}_{2}\right) g \omega_{2} \mathrm{Be}^{-\mathrm{i} \omega_{2} \tau}\right\}
\end{aligned}
$$

Introducing the polar notation $\mathrm{A}\left(\mathrm{T}_{1}\right)=\frac{1}{2} \mathrm{a}\left(\mathrm{T}_{1}\right) \mathrm{e}^{\mathrm{i} \theta_{1}\left(\mathrm{~T}_{1}\right)}$ and $\mathrm{B}\left(\mathrm{T}_{1}\right)=\frac{1}{2} \mathrm{~b}\left(\mathrm{~T}_{1}\right) \mathrm{e}^{\mathrm{i} \theta_{2}\left(\mathrm{~T}_{1}\right)}$ into Eqs. (16) $-(17)$ and setting the coefficients of the real and imaginary parts to zero yield the algebraic equations as

$$
\begin{aligned}
& \mathrm{a}^{\prime}=\frac{1}{\left(\mathrm{~m}_{1}+\mathrm{r}_{1}^{2} \mathrm{~m}_{2}\right) \omega_{1}}\left\{-\frac{\mathrm{a} \omega_{1}}{2}\left[\mathrm{c}_{1}-2 \mathrm{r}_{1} \mathrm{c}_{1}+\mathrm{r}_{1}^{2}\left(\mathrm{c}_{1}+\mathrm{c}_{2}\right)\right]+\frac{3 \mathrm{a}^{2} \mathrm{~b}}{8} \delta_{1}\left(1-\mathrm{r}_{1}\right)^{2}\left(1-\mathrm{r}_{2}\right) \sin \phi_{1}\right. \\
& \left.-\frac{\mathrm{a}}{2}\left(1-\mathrm{r}_{1}\right) g \omega_{1} \cos \left(\omega_{1} \tau\right)+\frac{1}{2} \mathrm{r}_{1} \mathrm{f} \sin \phi_{2}\right\} \\
& \mathrm{a} \phi_{2}^{\prime}=\frac{1}{\left(\mathrm{~m}_{1}+\mathrm{r}_{1}^{2} \mathrm{~m}_{2}\right) \omega_{1}}\left\{\left(\mathrm{~m}_{1}+\mathrm{r}_{1}^{2} \mathrm{~m}_{2}\right) \omega_{1} \mathrm{a} \sigma_{1}-\frac{3 a b^{2}}{4} \delta_{1}\left(1-\mathrm{r}_{1}\right)^{2}\left(1-\mathrm{r}_{2}\right)^{2}-\frac{3 \mathrm{a}^{3}}{8} \delta_{1}\left(1-\mathrm{r}_{1}\right)^{4}\right. \\
& \left.-\frac{3 \mathrm{a}^{2} \mathrm{~b}}{8} \delta_{1}\left(1-\mathrm{r}_{1}\right)^{2}\left(1-\mathrm{r}_{2}\right) \cos \phi_{1}-\frac{\mathrm{a}}{2}\left(1-\mathrm{r}_{1}\right) g \omega_{1} \sin \left(\omega_{1} \tau\right)+\frac{1}{2} \mathrm{r}_{1} \mathrm{f} \cos \phi_{2}\right\} \\
& \mathrm{b}^{\prime}=\frac{1}{\left(\mathrm{~m}_{1}+\mathrm{r}_{2}^{2} \mathrm{~m}_{2}\right) \omega_{2}}\left\{-\frac{\mathrm{b} \omega_{2}}{2}\left[\mathrm{c}_{1}-2 \mathrm{r}_{2} \mathrm{c}_{1}+\mathrm{r}_{2}^{2}\left(\mathrm{c}_{1}+\mathrm{c}_{2}\right)\right]-\frac{3 \mathrm{a}^{3}}{8} \delta_{1}\left(1-\mathrm{r}_{1}\right)^{3}\left(1-\mathrm{r}_{2}\right) \sin \phi_{1}\right. \\
& \left.-\frac{\mathrm{b}}{2}\left(1-\mathrm{r}_{2}\right) g \omega_{2} \cos \left(\omega_{2} \tau\right)\right\} \\
& \mathrm{b}\left(\phi_{1}^{\prime}+3 \phi_{2}^{\prime}\right)=\frac{1}{\left(\mathrm{~m}_{1}+\mathrm{r}_{2}^{2} \mathrm{~m}_{2}\right) \omega_{2}}\left\{3\left(\mathrm{~m}_{1}+\mathrm{r}_{2}^{2} \mathrm{~m}_{2}\right) \omega_{2} \mathrm{~b}\left(\sigma_{1}-\sigma_{3}\right)-\frac{3 \mathrm{a}^{2} \mathrm{~b}}{4} \delta_{1}\left(1-\mathrm{r}_{1}\right)^{2}\left(1-\mathrm{r}_{2}\right)^{2}\right. \\
& \left.-\frac{3 \mathrm{~b}^{3}}{8} \delta_{1}\left(1-\mathrm{r}_{2}\right)^{4}-\frac{3 \mathrm{a}^{3}}{8} \delta_{1}\left(1-\mathrm{r}_{1}\right)^{3}\left(1-\mathrm{r}_{2}\right) \cos \phi_{1}-\frac{\mathrm{b}}{2}\left(1-\mathrm{r}_{2}\right) g \omega_{2} \sin \left(\omega_{2} \tau\right)\right\}
\end{aligned}
$$

Where $\mathrm{a}$ and $\mathrm{b}$ are the amplitude of the first and second mode, $\phi_{1}=3 \theta_{1}-\theta_{2}-\sigma_{3} \mathrm{~T}_{1}, \phi_{2}=\sigma_{1} \mathrm{~T}_{1}-\theta_{1}$. The response of the absorber and primary system can be approximately express as follow: $\mathrm{y}_{1} \approx \mathrm{a} \cos \left[\omega_{1} \mathrm{~T}_{0}+\theta_{1}\left(\mathrm{~T}_{1}\right)\right]+\mathrm{b} \cos \left[\omega_{2} \mathrm{~T}_{0}+\theta_{2}\left(\mathrm{~T}_{1}\right)\right], \mathrm{y}_{2} \approx \mathrm{r}_{1} \mathrm{a} \cos \left[\omega_{1} \mathrm{~T}_{0}+\theta_{1}\left(\mathrm{~T}_{1}\right)\right]+\mathrm{r}_{2} \mathrm{~b} \cos \left[\omega_{2} \mathrm{~T}_{0}+\theta_{2}\left(\mathrm{~T}_{1}\right)\right]$

\section{The Effects of Delayed Feedback Control on Amplitude - Frequency Response Curves $\left(\omega \rightarrow \omega_{1}\right)$}

The equilibrium solutions can be obtained by setting $\mathrm{a}^{\prime}=\phi_{1}^{\prime}=\mathrm{b}=\phi_{2}^{\prime}$ in Eqs. (18)-(21). The detail process is omitted due to space limitations. The parameters are chosen as follows: 


$$
\begin{aligned}
& \mathrm{m}_{1}=6500 \mathrm{~kg}, \mathrm{~m}_{2}=1248 \mathrm{~kg}, \mathrm{k}_{1}=1.2 \times 10^{6} \mathrm{~N} / \mathrm{m}, \mathrm{k}_{2}=2.1 \times 10^{6} \mathrm{KN} / \mathrm{m}, \mathrm{c}_{1}=20 \mathrm{KN} \cdot \mathrm{s} / \mathrm{m}, \\
& \mathrm{c}_{2}=1.2 \mathrm{KN} \cdot \mathrm{s} / \mathrm{m}, \mathrm{f}=63520 \mathrm{~N} .
\end{aligned}
$$

Fig. 2-Fig. 10 show the amplitude-delay response curves of landing gear structure with different values of gain and external detuning parameters, where $y_{1}$ represents the amplitude of landing gear structure.

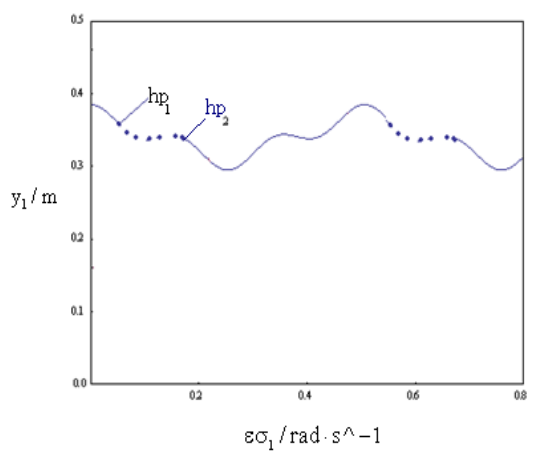

Fig. $2 \varepsilon \sigma_{1}=0, \mathrm{~g}=500 \mathrm{~N} . \mathrm{s} / \mathrm{m}$

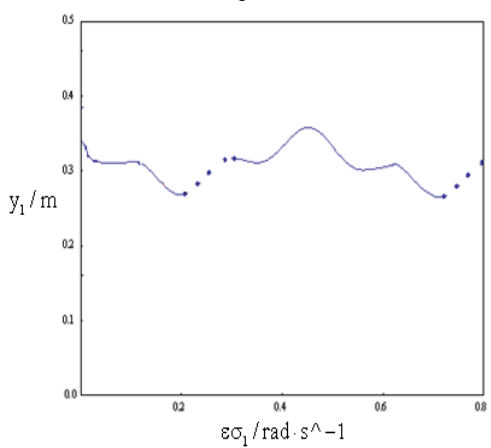

Fig. $5 \varepsilon \sigma_{1}=-0.3, \mathrm{~g}=500 \mathrm{~N} . \mathrm{s} / \mathrm{m}$

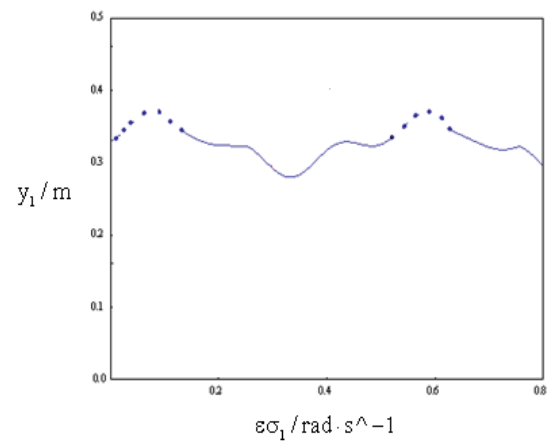

Fig. $8 \varepsilon \sigma_{1}=0.3, \mathrm{~g}=500 \mathrm{~N} . \mathrm{s} / \mathrm{m}$

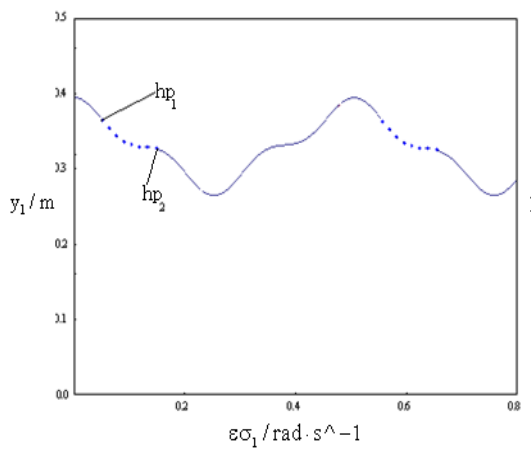

Fig. $3 \varepsilon \sigma_{1}=0, g=2000$ N.s $/ \mathrm{m}$

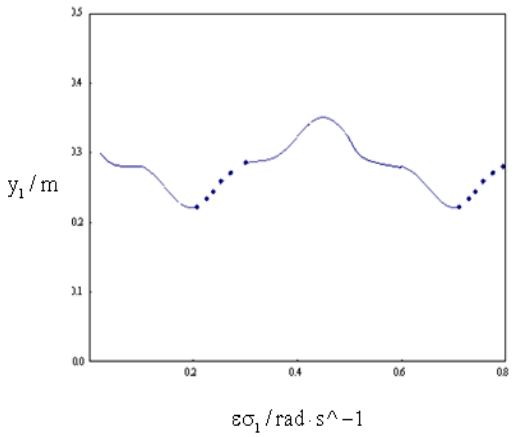

Fig. $6 \varepsilon \sigma_{1}=-0.3, g=2000$ N.s $/ \mathrm{m}$

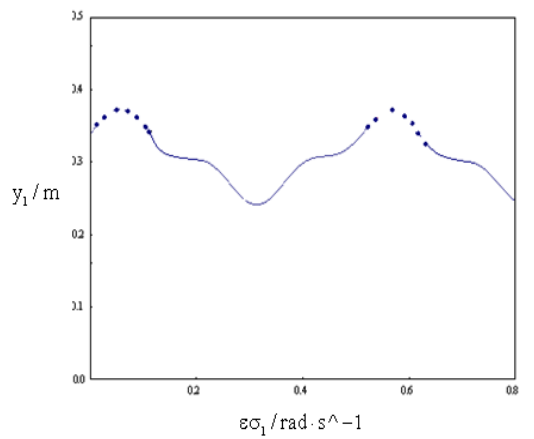

Fig. $9 \varepsilon \sigma_{1}=0.3, \mathrm{~g}=2000 \mathrm{~N} . \mathrm{s} / \mathrm{m}$

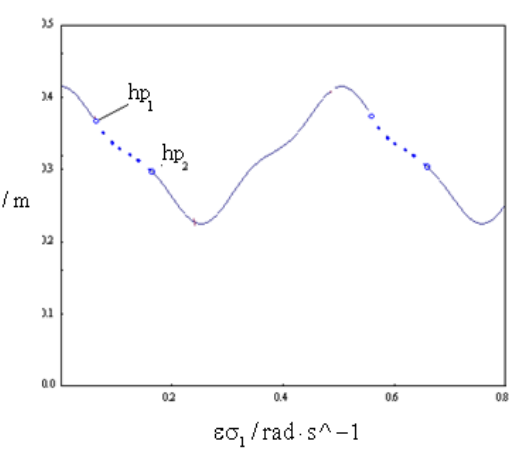

Fig. $4 \varepsilon \sigma_{1}=0, \mathrm{~g}=5000 \mathrm{~N} . \mathrm{s} / \mathrm{m}$

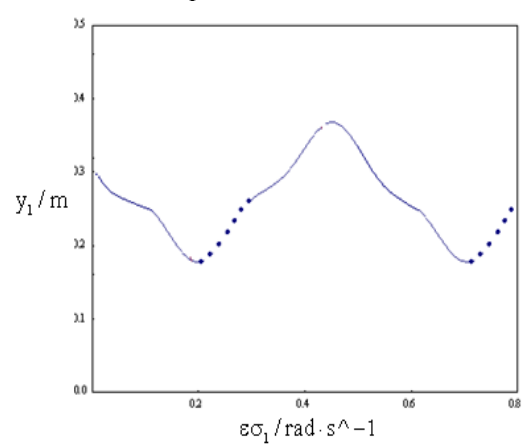

Fig. $7 \varepsilon \sigma_{1}=-0.3, g=5000 \mathrm{~N} . \mathrm{s} / \mathrm{m}$

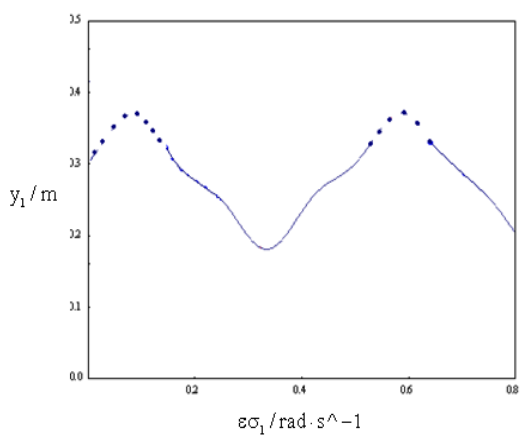

Fig. $10 \varepsilon \sigma_{1}=0.3, \mathrm{~g}=5000 \mathrm{~N} . \mathrm{s} / \mathrm{m}$

From Fig. 2-Fig. 7, it can be observed that as $\tau$ increases, the amplitude of landing gear structure increases in the beginning. Then the amplitude of it decreases as $\tau$ increases. Fig. 8-Fig. 10 show that as $\tau$ increases, the amplitude of landing gear structure decreases then increases. The effect of time delay on amplitude of landing gear structure is periodically. It can be observed that there are some interval for time delay the amplitude of the landing gear structure can be suppressed to a minimum value with different gain of time delay feedback. These time delay interval we called it "vibration suppression time delay interval". However, the system will lose its stability at other interval of time delay. These time delay interval we called it "unstable time delay interval".

Form Fig. 2-Fig. 10, we observe that as the gain of delayed feedback increases, the minimum value of amplitude of landing gear structure is decreased, it means the ability of vibration suppression is improved. However, as the gain of delayed feedback increases the unstable range of time delay is increases, it means the stability of the system is decreased. 
The Effects of Delayed Feedback Control on Amplitude - Frequency Response Curves $\left(\omega \rightarrow \omega_{2}\right)$

For the second case of $\omega \rightarrow \omega_{2}$, it can be obtained $\omega=\omega_{1}+\varepsilon \sigma_{1}, \omega_{2}=3 \omega_{1}+\varepsilon \sigma_{3}$. Setting the coefficients of the secular terms to zero into Eqs. (14)-(15), and yield the solvability conditions which are given by

The average equations are as follow.

$$
\begin{aligned}
& \mathrm{D}_{1} \mathrm{~A}=\frac{1}{2\left(\mathrm{~m}_{1}+\mathrm{r}_{1}^{2} \mathrm{~m}_{2}\right) i \omega_{1}}\left\{-\left[\mathrm{c}_{1}-2 \mathrm{r}_{1} \mathrm{c}_{1}+\mathrm{r}_{1}^{2}\left(\mathrm{c}_{1}+\mathrm{c}_{2}\right)\right] i \omega_{1} \mathrm{~A}-6 \delta_{1}\left(1-\mathrm{r}_{1}\right)^{2}\left(1-\mathrm{r}_{2}\right)^{2} \mathrm{~B} \overline{\mathrm{B}} \mathrm{A}\right. \\
& \left.-3 \delta_{1}\left(1-\mathrm{r}_{1}\right)^{4} \mathrm{~A}^{2} \overline{\mathrm{A}}-3 \delta_{1}\left(1-\mathrm{r}_{1}\right)^{3}\left(1-\mathrm{r}_{2}\right) \overline{\mathrm{A}}^{2} \mathrm{Be}^{\mathrm{i} \sigma_{3} \mathrm{~T}_{1}}-\mathrm{i}\left(1-\mathrm{r}_{1}\right) g \omega_{1} A \mathrm{e}^{-\mathrm{i} \omega_{1} \tau}\right\} \\
& \mathrm{D}_{1} \mathrm{~B}=\frac{1}{2\left(\mathrm{~m}_{1}+\mathrm{r}_{2}^{2} \mathrm{~m}_{2}\right) i \omega_{2}}\left\{-\left[\mathrm{c}_{1}-2 \mathrm{r}_{2} \mathrm{c}_{1}+\mathrm{r}_{2}^{2}\left(\mathrm{c}_{1}+\mathrm{c}_{2}\right)\right] i \omega_{2} \mathrm{~B}-6 \delta_{1}\left(1-\mathrm{r}_{2}\right)^{2}\left(1-\mathrm{r}_{1}\right)^{2} \mathrm{~A} \overline{\mathrm{A}} \mathrm{B}\right. \\
& \left.-3 \delta_{1}\left(1-\mathrm{r}_{2}\right)^{4} \mathrm{~B}^{2} \overline{\mathrm{B}}-\delta_{1}\left(1-\mathrm{r}_{1}\right)^{3}\left(1-\mathrm{r}_{2}\right) \mathrm{A}^{3} \mathrm{e}^{-\mathrm{i} \sigma_{3} \mathrm{~T}_{1}}-\mathrm{i}\left(1-\mathrm{r}_{2}\right) g \omega_{2} \mathrm{Be}^{-i \omega_{2} \tau}+\frac{1}{2} \mathrm{r}_{2} \mathrm{fe}^{\mathrm{i} \mathrm{\sigma}_{1} \mathrm{~T}_{1}}\right\}
\end{aligned}
$$

Introducing the polar notation $\mathrm{A}\left(\mathrm{T}_{1}\right)=\frac{1}{2} \mathrm{a}\left(\mathrm{T}_{1}\right) \mathrm{e}^{\mathrm{i} \theta_{1}\left(\mathrm{~T}_{1}\right)}$ and $\mathrm{B}\left(\mathrm{T}_{1}\right)=\frac{1}{2} \mathrm{~b}\left(\mathrm{~T}_{1}\right) \mathrm{e}^{\mathrm{i} \theta_{2}\left(\mathrm{~T}_{1}\right)}$ into Eqs. (22) $-(23)$ and setting the coefficients of the real and imaginary parts to zero yield the algebraic equations as

$$
\begin{aligned}
& \mathrm{a}^{\prime}=\frac{1}{\left(\mathrm{~m}_{1}+\mathrm{r}_{1}^{2} \mathrm{~m}_{2}\right) \omega_{1}}\left\{-\frac{\mathrm{a} \omega_{1}}{2}\left[\mathrm{c}_{1}-2 \mathrm{r}_{1} \mathrm{c}_{1}+\mathrm{r}_{1}^{2}\left(\mathrm{c}_{1}+\mathrm{c}_{2}\right)\right]+\frac{3 \mathrm{a}^{2} \mathrm{~b}}{8} \delta_{1}\left(1-\mathrm{r}_{1}\right)^{2}\left(1-\mathrm{r}_{2}\right) \sin \phi_{1}\right. \\
& \left.-\frac{\mathrm{a}}{2}\left(1-\mathrm{r}_{1}\right) g \omega_{1} \cos \left(\omega_{1} \tau\right)\right\} \\
& \mathrm{a}\left(\frac{\phi_{1}^{\prime}-\phi_{2}^{\prime}}{3}\right)=\frac{1}{\left(\mathrm{~m}_{1}+\mathrm{r}_{1}^{2} \mathrm{~m}_{2}\right) \omega_{1}}\left\{\left(\mathrm{~m}_{1}+\mathrm{r}_{1}^{2} \mathrm{~m}_{2}\right) \omega_{1} \mathrm{a}\left(\frac{\sigma_{1}+\sigma_{3}}{3}\right)+\frac{3 a b^{2}}{4} \delta_{1}\left(1-\mathrm{r}_{1}\right)^{2}\left(1-\mathrm{r}_{2}\right)^{2}\right. \\
& \left.+\frac{3 \mathrm{a}^{3}}{8} \delta_{1}\left(1-\mathrm{r}_{1}\right)^{4}+\frac{3 \mathrm{a}^{2} \mathrm{~b}}{8} \delta_{1}\left(1-\mathrm{r}_{1}\right)^{2}\left(1-\mathrm{r}_{2}\right) \cos \phi_{1}+\frac{\mathrm{a}}{2}\left(1-\mathrm{r}_{1}\right) g \omega_{1} \sin \left(\omega_{1} \tau\right)\right\} \\
& \mathrm{b}^{\prime}=\frac{1}{\left(\mathrm{~m}_{1}+\mathrm{r}_{2}^{2} \mathrm{~m}_{2}\right) \omega_{2}}\left\{-\frac{\mathrm{b} \omega_{2}}{2}\left[\mathrm{c}_{1}-2 \mathrm{r}_{2} \mathrm{c}_{1}+\mathrm{r}_{2}^{2}\left(\mathrm{c}_{1}+\mathrm{c}_{2}\right)\right]-\frac{3 \mathrm{a}^{3}}{8} \delta_{1}\left(1-\mathrm{r}_{1}\right)^{3}\left(1-\mathrm{r}_{2}\right) \sin \phi_{1}\right. \\
& \left.-\frac{\mathrm{b}}{2}\left(1-\mathrm{r}_{2}\right) g \omega_{2} \cos \left(\omega_{2} \tau\right)+\frac{1}{2} \mathrm{r}_{2} \mathrm{f} \sin \phi_{2}\right\} \\
& \mathrm{b}\left(\phi_{2}^{\prime}\right)=\frac{1}{\left(\mathrm{~m}_{1}+\mathrm{r}_{2}^{2} \mathrm{~m}_{2}\right) \omega_{2}}\left\{\left(\mathrm{~m}_{1}+\mathrm{r}_{2}^{2} \mathrm{~m}_{2}\right) \omega_{2} \mathrm{~b} \sigma_{1}-\frac{3 \mathrm{a}^{2} \mathrm{~b}}{4} \delta_{1}\left(1-\mathrm{r}_{1}\right)^{2}\left(1-\mathrm{r}_{2}\right)^{2}-\frac{3 \mathrm{~b}^{3}}{8}\right. \\
& \left.\delta_{1}\left(1-\mathrm{r}_{2}\right)^{4}-\frac{3 \mathrm{a}^{3}}{8} \delta_{1}\left(1-\mathrm{r}_{1}\right)^{3}\left(1-\mathrm{r}_{2}\right) \cos \phi_{1}-\frac{\mathrm{b}}{2}\left(1-\mathrm{r}_{2}\right) g \omega_{2} \sin \left(\omega_{2} \tau\right)+\frac{1}{2} \mathrm{r}_{2} \mathrm{f} \cos \phi_{2}\right\}
\end{aligned}
$$

where $\phi_{1}=3 \theta_{1}-\theta_{2}-\sigma_{3} \mathrm{~T}_{1}, \phi_{2}=\sigma_{1} \mathrm{~T}_{1}-\theta_{2}$.

Fig. 11-Fig. 13 show the amplitude-delay response curves of landing gear structure with a fixed gain and different values of external detuning parameters, where $\mathrm{y}_{1}$ represents the amplitude of landing gear structure. 

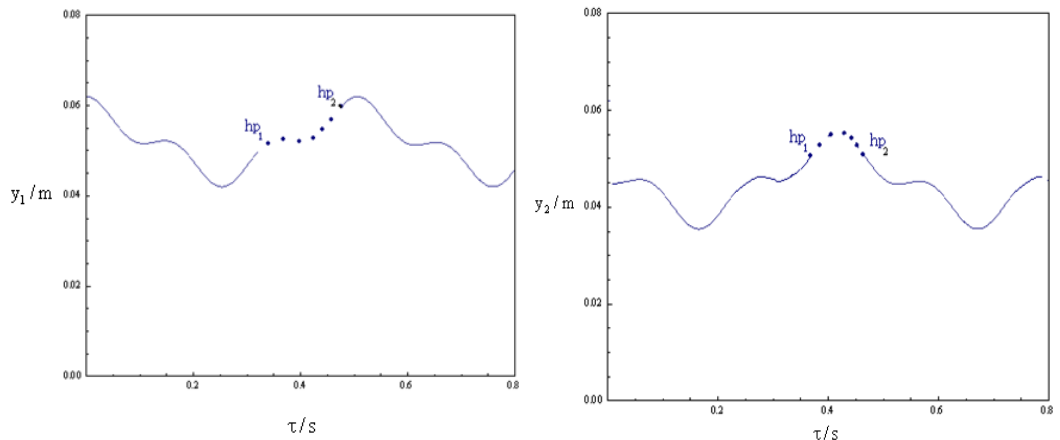

Fig. $11 \varepsilon \sigma_{1}=0, \mathrm{~g}=3000 \mathrm{~N} . \mathrm{s} / \mathrm{m} \quad$ Fig. $12 \varepsilon \sigma_{1}=-0.3, \mathrm{~g}=3000 \mathrm{~N} . \mathrm{s} / \mathrm{m}$

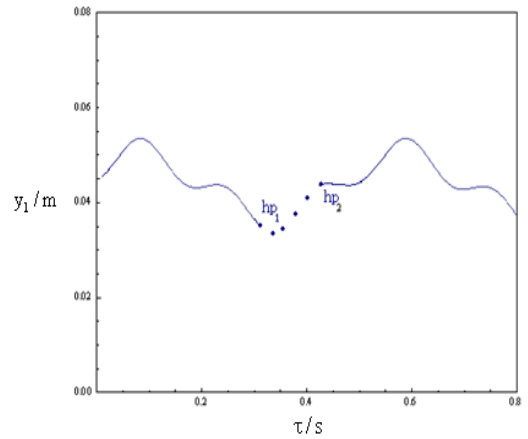

Fig. $13 \varepsilon \sigma_{1}=0.3, g=5000$ N.s $/ \mathrm{m}$

Fig. 11-Fig. 13 show that the amplitude-delay response curves of landing gear structure is affected by internal detuning parameters, the response curve shifts left and right with the variation of internal detuning parameters.

\section{Numerical Simulation}

In the following parts, we give some examples of numerical simulations to prove the above analytical results in quantitatively. Fig. 14 - Fig. 17 show the time history response curves of landing gear with different gain and time delay.

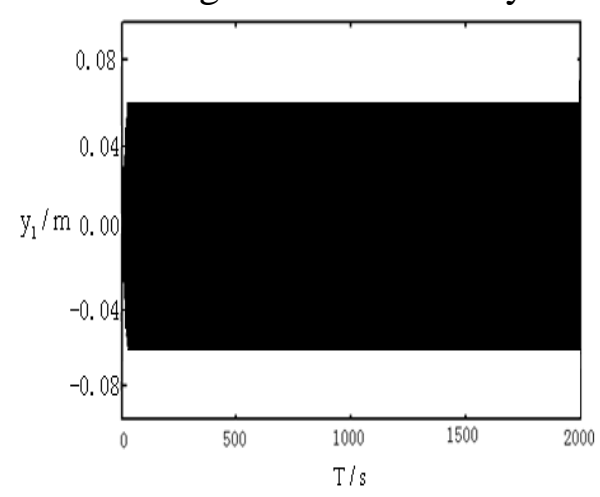

Fig. 14: Time history response curve $(\mathrm{g}=0, \tau=0)$

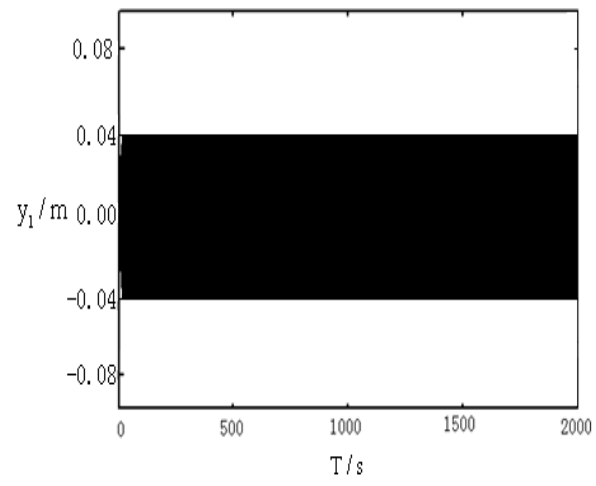

Fig. 16: Time history response curve ( $\mathrm{g}=3000 \mathrm{~N} . \mathrm{s} / \mathrm{m}, \tau=0.27 \mathrm{~s})$

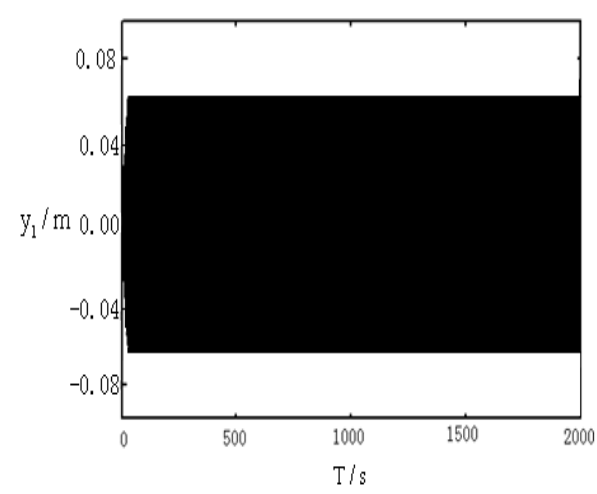

Fig. 15: Time history response curve ( $\mathrm{g}=3000 \mathrm{~N} . \mathrm{s} / \mathrm{m}, \tau=0)$

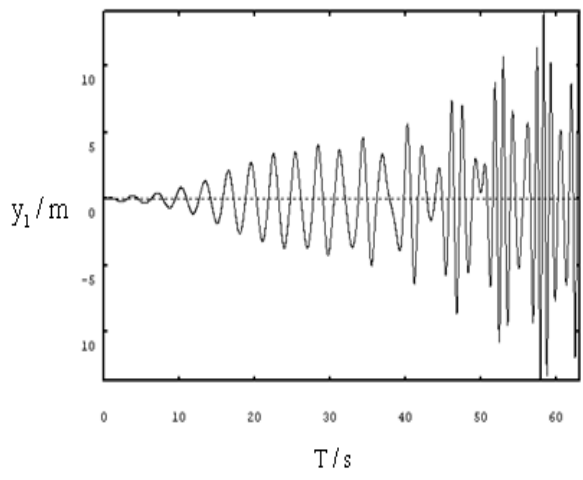

Fig. 17: Time history response curve ( $\mathrm{g}=3000$ N.s $/ \mathrm{m}, \tau=0.4 \mathrm{~s}$ )

Fig. 14 shows the passive control system. Fig. 15 shows that if the feedback control without time delay, the vibration of the landing gear system is increased. Fig. 16 shows that if the gain and time delay are appropriate chosen, the vibration of the system is suppressed well. Fig. 17 shows that if the gain and time delay are not appropriate, the landing gear system is unstable. 


\section{Summary}

In the present paper, a delayed feedback control is employed to control the vibration in landing gear structure. When the system is in 1:3 internal resonance, the dynamical behavior of the system is complex. We investigate two cases which the external excitation frequency nearing to first and second mode frequency. The effects of gain and time delay on vibration suppression of landing gear system are analyzed. It can be observed that the ability of vibration suppression is improved when the gain is increased. However, the unstable range is enlarged when the gain is increased. The effect of time delay on amplitude of landing gear structure is periodically. It means that if we choose appropriate values of time delay, the vibration of the landing gear structure can be suppressed to a minimum value.

\section{Acknowledgement}

This research was financially supported by the National Natural Science Foundation of China No. 11372125, Natural Science Foundation of Jiangxi Province No. 20161BAB101005 ,Project of Education Department of Jiangxi Province No. DB201606020.

\section{References}

[1] F. Michael. Theoretical and experimental principles of landing gear research and development. Luftfuhrt Forschung. 14 (1937) 387-416.

[2] K. Schlaefke. On force-defection diagrams of shock struts T B. 11(1944).

[3] W. Kochanowsky. Landing and taxing impacts on oil shock struts. Deutsche Luftfahr forschung, 32 (1944) 465-473.

[4] E. Marquard, W. Meyerzur Capellen. Approximate calculation of the force between landing gear and fuselage of a landing Aircraft. Symmetric Landing Forschung Sbericht Nr 173711 Teach H S Aachen, 18 (1943) 225-231.

[5] W. Flugge. The influence of wheel spin-up on landing gear impact. NACA-TN-3217, (10) 1954 01-09.

[6] Y. T. Choi, N. M. Wereley. Vibration control of a landing gear system featuring electrorheology / Magetorheological Fluids. Journal of Aircraft. 40 (2003) 432-439. 